\section{Lobar collapse due to endobronchial metastatic prostatic carcinoma: re-expansion with antiandrogen treatment}

\author{
Helena Taylor, Stanley Braude
}

\begin{abstract}
A patient is described who presented with left upper lobe collapse due to endobronchial metastatic prostate carcinoma. Treatment with the oral antiandrogen cyproterone acetate resulted in resolution of the occluding endobronchial carcinoma and lobar re-expansion.
\end{abstract}

Pulmonary metastases from prostatic carcinoma are rarely recognised clinically. ${ }^{1}$ The most common radiological pattern of pulmonary metastases is discrete tumour nodules ${ }^{2}$; lymphangitis carcinomatosa and pleural effusions are seen less frequently. Isolated reports suggest that these manifestations of metastatic prostatic carcinoma may respond to

Department of Thoracic Medicine, King's College Hospital, London Helena Taylor Stanley Braude

Address for reprint requests Dr $S$ Braude, Brompton Hospital, London SW3 6HP

Accepted 3 October 1989 re-expanded. appropriate hormonal treatment. ${ }^{34}$ Endobronchial metastases, which may simulate bronchogenic carcinoma, have been reported only once $^{5}$ and their response to endocrine manipulation has not been documented. We report a case of widespread endobronchial metastases from prostatic carcinoma, where the initial presentation with lobar collapse was highly suggestive of a primary bronchogenic carcinoma. Antiandrogen treatment produced a prompt improvement in symptoms and in the radiological and bronchoscopic appearances.

\section{Case report}

An 81 year old man presented in April 1987 with symptoms of prostatism. Prostatic biopsy showed a well differentiated adenocarcinoma and he underwent a transurethral resection. The acid phosphatase activity was 7.9 (normal $<4.5) \mathrm{IU} / 1$ and a bone scan was normal. At that time he had no respiratory symptoms and the chest radiograph was normal.

On review in October 1988 he gave a six month history of progressive breathlessness and occasional streaky haemoptyses. He had been a lifelong heavy smoker. Clinically and radiologically there were signs of left upper lobe collapse (figure, A). The peak expiratory flow (PEF) was $140 \mathrm{l} / \mathrm{min}$. A bone scan showed numerous areas of increased uptake, particularly in the dorsolumbar spine, though he had not experienced pain. The acid phosphatase was $40.6 \mathrm{IU} / 1$. Fibreoptic bronchoscopy showed the mucosa of the distal trachea, the main carina, and the right main and intermediate bronchus to be diffusely affected by endobronchial carcinoma, though without reduction in calibre. The left main bronchus was substantially narrowed throughout its length by endobronchial tumour, and the left upper lobe orifice was completely occluded by

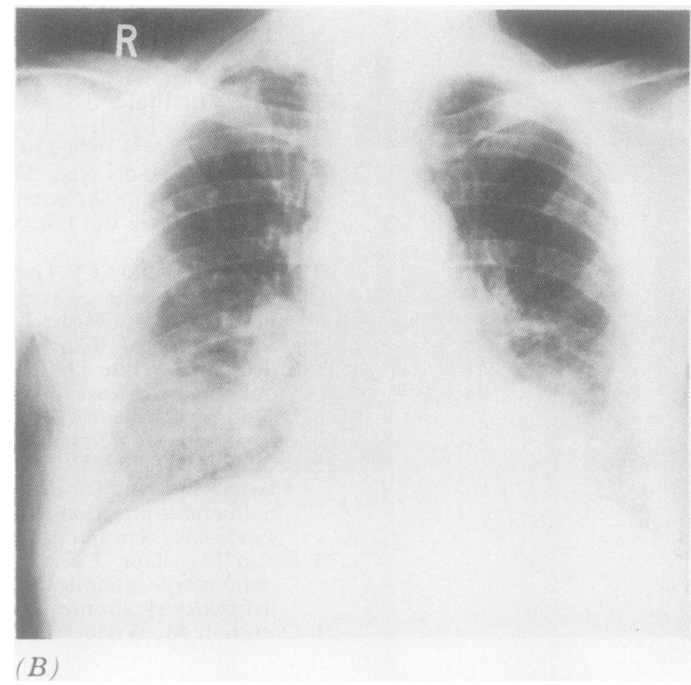

Posteroanterior chest radiographs at presentation, $(A)$ showing a prominent right hilum, left upper lobe collapse, and lower zone shadowing consistent with lymphangitis. After six weeks' antiandrogen treatment (B) the left upper lobe has 
polypoid tumour. Histological examination of endobronchial biopsy specimens showed moderately differentiated adenocarcinoma of a microacinar pattern. Immunocytochemical studies showed positive staining when prostatic acid phosphatase antibody was used. Antiandrogen treatment, cyproterone acetate $100 \mathrm{mg}$ thrice daily, was started.

After six weeks' treatment his breathlessness had improved and he had had no further haemoptyses. His PEF was $205 \mathrm{l} / \mathrm{min}$. The chest radiograph showed re-expansion of the left upper lobe (figure, B). Bronchoscopically there was evidence of dramatic regression of the extensive endobronchial carcinoma previously noted. The left upper lobe orifice was completely patent, though isolated tumour nodules remained in both main bronchi. The left main bronchus was now of normal calibre.

\section{Discussion}

Despite its infrequent clinical recognition, necropsy analysis suggests that pulmonary metastases from prostatic carcinoma are relatively common, occurring in up to a quarter of cases. ${ }^{1}$ Diagnostic problems may arise when the primary prostatic tumour is not known, or if the pulmonary presentation suggests a bronchogenic carcinoma. This case shows the importance of differentiation from primary bronchogenic adenocarcinoma, especially in view of the dramatic response to hormonal treatment.

Endobronchial metastatic disease is well recognised, particularly in patients with breast, renal, and colonic carcinoma. ${ }^{6}$ Extensive bilateral endobronchial metastases, as in this patient, however, have been documented only once previously in prostatic carcinoma. ${ }^{5}$ Lobar collapse and subsequent resolution after antiandrogen treatment has not been recorded. The diffuseness of endobronchial tumour in this patient, without obvious parenchymal abnormalities radiologically, suggests that these were primarily endobronchial metastases.

Elderly men presenting with an apparent primary bronchogenic adenocarcinoma should, therefore, have the diagnosis of metastatic prostate cancer actively considered. Clinical examination and estimation of serum acid phosphatase should be part of the diagnostic routine in this group. Immunocytochemical studies of the bronchoscopic biopsy specimens may establish conclusively, as in this patient, the metastatic nature of the lesion. Appropriate treatment may result in substantial symptomatic and objective improvement and may prolong survival.

We thank Dr Frances Calman for her helpful advice about management.

1 Bolton BH. Pulmonary metastases from carcinoma of the prostate: incidence and case report of a long remission. $J$ Urol 1965;94:73-7.

2 Keeping IM, Buchanan R, Dadds JH. Microscopic tumour emboli from carcinoma of the prostate. Br J Dis Chest 1982;76:289-300.

3 Morin ME, MacNealy GA, Tan A, Li Y-P, Engel G, Hennebury $\mathbf{M}$. Hormonal manipulation of pulmonary metastases from carcinoma of the prostate. Urol Radiol 1982;4:23-7.

4 Heffner JE, Duffey DJ, Schwarz MI. Massive pleural effusions from prostatic lymphangitic carcinomatosis. Resolution with endocrine therapy. Arch Intern Med 1982;142:375-6.

5 Lalli C, Gogia H, Raju L. Multiple endobronchial metastases from carcinoma of prostate. Urology 1983;21:164-5.

6 Shepard MP. Endobronchial metastatic disease. Thorax 1982;37:362-5. 